\title{
The isolated scattering number can be computed in polynomial time for interval graphs
}

\author{
Fengwei $\mathrm{Li}^{1} \quad$ Qingfang $\mathrm{Ye}^{2} \quad$ Yuefang $\mathrm{Sun}^{3}$
}

(Received 13 June 2016; revised 26 January 2017)

\begin{abstract}
The isolated scattering number of an incomplete connected graph $G$ is defined as $\operatorname{isc}(G)=\max \{i(G-X)-|X|: X \in C(G)\}$, where $i(G-X)$ and $\mathrm{C}(\mathrm{G})$, respectively, denote the number of components which are isolated vertices and the set of all separators of G. The isolated scattering number is a comparatively better parameter to measure the vulnerability of networks. We give a polynomial time algorithm to compute the isolated scattering number of interval graphs, a subclass of co-comparability graphs. Our result can also be used to compute isolated scattering number of proper interval graph.
\end{abstract}

DOI:10.21914/anziamj.v58i0.10993, (C) Austral. Mathematical Soc. 2017. Published March 13, 2017, as part of the Proceedings of the 2016 Joint Conference of ANZIAM and Zhejiang Provincial Applied Mathematics Association. ISSN 1445-8810. (Print two pages per sheet of paper.) Copies of this article must not be made otherwise available on the internet; instead link directly to the DOI for this article. Record comments on this article via

http://journal austms.org.au/ojs/index.php/ANZIAMJ/comment/add/10993/0 
Subject class: 90B18, 94C15, 05C40,05C90

Keywords: Isolated scattering number, Vulnerability, Interval graph, Consecutive clique arrangement, Polynomial time algorithm.

\section{Contents}

1 Introduction

E82

2 Preliminaries

E85

3 Isolated scattering number for interval graphs

E88

4 Conclusion

E93

References

E94

\section{Introduction}

A communication network is composed of processors and communication links. If the network begins losing communication links or processors, then there is a loss in its effectiveness. This event is called the vulnerability of communication networks.

The vulnerability of communication networks measures the resistance of a network to a disruption in operation after the failure of certain processors and communication links. Cable cuts, processor interruptions, software errors, hardware failures, or transmission failure at various points can interrupt service for a long period of time. But network designs require greater degrees of stability and reliability or less vulnerability in communication networks. Thus, communication networks must be constructed to be as stable as possible, 
not only with respect to the initial disruption, but also with respect to the possible reconfiguration of the network.

In an analysis of the vulnerability of networks to disruption, three important quantities (there may be others) are

1. the number of elements that are not functioning;

2. the number of remaining connected sub-networks; and

3. the size of a largest remaining group within which mutual communication can still occur.

A communication network is modelled as an undirected and unweighted graph, where a processor (station) is represented as a vertex and a communication link between processors (stations) as an edge between corresponding vertices. When we use a graph to model a network, based on the above three quantities, a number of graph parameters have been proposed for measuring the vulnerability of networks, such as connectivity, toughness [6], scattering number [11], integrity [1], tenacity [7], rupture degree [19], isolated rupture degree [16, 17] and their edge-analogues.

We only consider finite simple undirected graphs, and we refer to the textbook of Bondy and Murty [2] for any undefined graph terminology and notations. The vertex set of a graph $G$ is denoted by $V$ and the edge set of $G$ is denoted by $E$. For $X \subseteq V(G)$, let $\omega(G-X)$ and $i(G-X)$, respectively, denote the number of components, the number of components which are isolated vertices in $G-X$. If $X$ is a vertex subset of $V$, then we use $G[X]$ to denote the subgraph of $\mathrm{G}$ induced by $\mathrm{X}$. A subset $\mathrm{X} \subseteq \mathrm{V}$ is a separator (or cut-set) of a graph $G=(V, E)$ if $G-X$ has more than one component. The empty set $\emptyset$ is a separator of $G$ if and only if $G$ is disconnected. We let $C(G)$ denote the set of all separators of $\mathrm{G}$.

One of the vulnerability parameters is the scattering number which take into account the quantities 1 and 2. The scattering number was introduced by Jung [11] in 1978, and the scattering number of an incomplete connected 
graph $\mathrm{G}$ is defined as

$$
s(G)=\max \{\omega(G-S)-|S|: S \in C(G), \omega(G-S)>1\} .
$$

Motivated from Jung's scattering number by replacing $\omega(G-X)$ with $i(G-X)$ in the above definition, Wang et al. [21] introduced the isolated scattering number, isc (G), as a new parameter to measure the vulnerability of networks. Definition 1 ([21]). The isolated scattering number of an incomplete connected graph $\mathrm{G}$ is defined as

$$
\operatorname{isc}(G)=\max \{\mathfrak{i}(G-X)-|X|: X \in C(G)\},
$$

where the maximum is taken over all the separators of $\mathrm{G}$.

Since a complete graph $K_{n}$ has no separators, we define isc $\left(K_{n}\right)=2-n$.

Definition 2. A separator $\mathrm{X}$ of an incomplete connected graph $\mathrm{G}$ is called an isc-set of $\mathrm{G}$ if $\operatorname{isc}(\mathrm{G})=\mathfrak{i}(\mathrm{G}-\mathrm{X})-|\mathrm{X}|$.

The isolated scattering number is of particular interest because it is considered to be a reasonable measure for the vulnerability of graphs. As a new graph parameter to measure the vulnerability of networks, scattering number and isolated scattering number, differ in showing the vulnerability of networks. This can be shown as follows. Consider the graphs $G_{1}$ and $G_{2}$ in Figure 1: it is not difficult to check that $s\left(G_{1}\right)=s\left(G_{2}\right)=5$, but isc $\left(G_{1}\right)=1 \neq 5=\operatorname{isc}\left(G_{2}\right)$.

Hence, the isolated scattering number is a reasonable parameter for distinguishing the vulnerability of these graphs. The less the isolated scattering number of a network the more stable it is considered to be [16]. Wang et al. [21] gave formulas for the isolated scattering number of join graphs and some bounds of the isolated scattering number, and they also give a recursive algorithm for computing the isolated rupture degree of trees. We prove that for interval graphs this number can be computed in polynomial times. 
Figure 1: Two graphs with the same scattering number but the different isolated scattering number

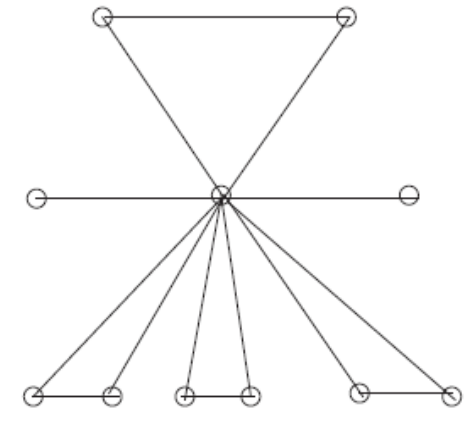

$G_{1}$

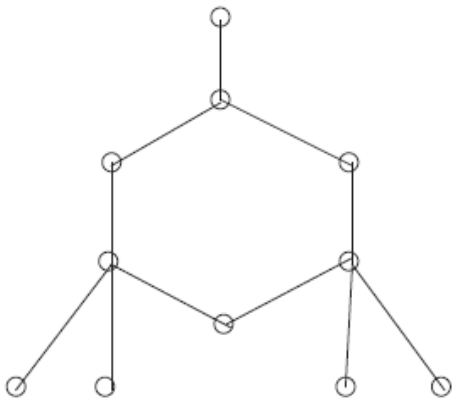

$G_{2}$

\section{Preliminaries}

This section characterises the property of minimal separator $X$ of a graph $G$, and gives a formula to calculate isolated scattering number via minimal separator $\mathrm{X}$ of a graph G. First, we define the minimal separator.

Definition 3 ([13]). A subset $\mathrm{X} \subseteq \mathrm{V}$ of $\mathrm{G}$ is called an a,b-separator for nonadjacent vertices $\mathrm{a}$ and $\mathrm{b}$ of graph $\mathrm{G}$ if the removal of $\mathrm{X}$ separates $\mathrm{a}$ and $\mathrm{b}$ in distinct connected components. If no proper subset of $\mathrm{X}$ is an $\mathrm{a}, \mathrm{b}$-separator of graph $\mathrm{G}$, then $\mathrm{X}$ is called a minimal a,b-separator of $\mathrm{G}$. A minimal separator $\mathrm{X}$ of $\mathrm{G}$ is a set of vertices such that $\mathrm{X}$ is a minimal $\mathrm{a}, \mathrm{b}$-separator for some nonadjacent vertices $\mathrm{a}$ and $\mathrm{b}$.

The following lemma provides an easy test of whether or not a given vertex set $X$ is a minimal separator [14].

Lemma 4 ([14]). Let $\mathrm{X}$ be a separator of the graph $\mathrm{G}=(\mathrm{V}, \mathrm{E})$. Then $\mathrm{X}$ is a minimal separator if and only if there are at least two different connected 
components of $\mathrm{G}-\mathrm{X}$ such that every vertex of $\mathrm{X}$ has a neighbour in both of these components.

Theorem 5. Let $\mathrm{G}$ be an incomplete graph. Then

$$
\operatorname{isc}(G)=\max _{X^{*}}\left\{\sum_{i=k+1}^{t} \max \left[\operatorname{isc}\left(G\left[C_{i}\right]\right), 0\right]-\left|X^{*}\right|+k\right\},
$$

where the maximum is taken over all minimal separators $\mathrm{X}^{*}$ of the graph $\mathrm{G}$, $\mathrm{C}_{1}, \mathrm{C}_{2}, \ldots, \mathrm{C}_{\mathrm{k}}$ are the components of $\mathrm{G}-\mathrm{X}^{*}$ which are isolated vertices, and $\mathrm{C}_{\mathrm{k}+1}, \mathrm{C}_{\mathrm{k}+2}, \ldots, \mathrm{C}_{\mathrm{t}}$ are the connected components of $\mathrm{G}-\mathrm{X}^{*}$ which are not isolated vertices.

Proof: First let $X$ be an isc-set with the minimum number of vertices among all isc-sets of $G$. Let $X^{*}$ be a minimal separator of $G$ that is a subset of $X$, we suppose $C_{1}, C_{2}, \ldots, C_{k}$ be the components of $G-X^{*}$ which are isolated vertices, and let $C_{k+1}, C_{k+2}, \ldots, C_{t}$ be the connected components of $G-X^{*}$ which are not isolated vertices. Then $C_{1}, C_{2}, \ldots, C_{k}$ are also the components of $G-X$, so, we consider the sets $X_{i}=X \cap C_{i}, i \in\{k+1, k+2, \ldots, t\}$. The proof proceeds in the following two cases.

1. $X_{i}=\emptyset$. Then, $C_{i}$ is also a component of $G-X$, and so $i\left(C_{i}-X_{i}\right)-\left|X_{i}\right|=$ 0 .

2. $X_{i} \neq \emptyset$, that is, $\left|X_{i}\right| \geqslant 1$. Suppose that $X_{i}$ is not a separator of $G\left[C_{i}\right]$. Then $i\left(G-\left(X-X_{i}\right)\right) \geqslant i(G-X)-1$.

(a) If $\left|X_{i}\right|=1$, then

$$
\begin{aligned}
i\left(G-\left(X-X_{i}\right)\right)-\left|X-X_{i}\right| & \geqslant i(G-X)-1-|X|+\left|X_{i}\right| \\
& \geqslant i(G-X)-|X|=\operatorname{isc}(G),
\end{aligned}
$$

that is, $\mathrm{X}-\mathrm{X}_{i}$ is also an isc-set of $\mathrm{G}$, a contradiction to the minimality of $X$. 
(b) If $\left|X_{i}\right|>1$, then

$$
\begin{aligned}
i\left(G-\left(X-X_{i}\right)\right)-\left|X-X_{i}\right| & \geqslant i(G-X)-1-|X|+\left|X_{i}\right| \\
& >i(G-X)-|X|=i \operatorname{sc}(G),
\end{aligned}
$$

a contradiction to the definition of isolated scattering number of graphs. Hence $X_{i} \neq \emptyset$ implies that $X_{i}$ is a separator of $G\left[C_{i}\right]$. Thus, isc $\left(G\left[C_{i}\right]\right) \geqslant i\left(G\left[C_{i}\right]-X_{i}\right)-\left|X_{i}\right|$.

Summing up the values of $i\left(G\left[C_{i}\right]-X_{i}\right)-\left|X_{i}\right|$ over all components $C_{i}$ of $G-X^{*}$ will achieve the value of $i(G-X)-|X|=\operatorname{isc}(G)$. Thus

$$
\begin{aligned}
\operatorname{isc}(G) & =\mathfrak{i}(G-X)-|X| \\
& =\sum_{i=k+1}^{t}\left\{i\left(G\left[C_{i}\right]-X_{i}\right)-\left|X_{i}\right|\right\}-\left|X^{*}\right|+k \\
& \leqslant \sum_{i=k+1}^{t} \max \left[\operatorname{isc}\left(G\left[C_{i}\right]\right), 0\right]-\left|X^{*}\right|+k .
\end{aligned}
$$

On the other hand, let $X^{*}$ be a minimal separator of $G$ with $\omega\left(G-X^{*}\right) \geqslant 2$. Furthermore let $C_{1}, C_{2}, \ldots, C_{k}$ be the components of $G-X^{*}$ which are isolated vertices, and let $C_{k+1}, C_{k+2}, \ldots, C_{t}$ be the connected components of $G-X^{*}$ which are not isolated vertices. Then we construct a separator of $G$ such that $X=X^{*} \cup\left(\cup_{i=k+1}^{t} X_{i}\right)$ with $X_{i} \subset C_{i}$ for every $i \in\{k+1, k+2, \ldots, t\}$. For $i \in\{k+1, k+2, \ldots, t\}$ we set $X_{i}=\emptyset$ if isc $\left(G\left[C_{i}\right]\right) \leqslant 0$. Otherwise, if $\operatorname{isc}\left(G\left[C_{i}\right]\right)>0$, we choose a separator $X_{i}$ of $G\left[C_{i}\right]$ such that isc $\left(G\left[C_{i}\right]\right)=$ $i\left(G\left[C_{i}\right]-X_{i}\right)-\left|X_{i}\right|$. Then $X \supset X^{*}$ is a separator of $G$ and so

$$
\operatorname{isc}(G) \geqslant i(G-X)-|X|=\sum_{i=k+1}^{t}\left\{i\left(G\left[C_{i}\right]-X_{i}\right)-\left|X_{i}\right|\right\}-\left|X^{*}\right|+k .
$$


Without loss of generality, let $C_{k+1}, C_{k+2}, \ldots, C_{k+r}, 0 \leqslant r \leqslant t-k$, be the connected components of $\mathrm{G}$ with isc $\left(\mathrm{G}\left[\mathrm{C}_{i}\right]\right) \leqslant 0$. Consequently,

$$
\begin{aligned}
\operatorname{isc}(G) & \geqslant \sum_{i=k+1}^{t}\left\{i\left(G\left[C_{i}\right]-X_{i}\right)-\left|X_{i}\right|\right\}-\left|X^{*}\right|+k \\
& =\sum_{i=k+1}^{k+r} 0+\sum_{i=k+r+1}^{t}\left(\operatorname{isc}\left(G\left[C_{i}\right]\right)-\left|X^{*}\right|\right)+k \\
& \geqslant \sum_{i=k+1}^{t} \max \left[\operatorname{isc}\left(G\left[C_{i}\right]\right), 0\right]-\left|X^{*}\right|+k .
\end{aligned}
$$

This completes the proof.

\section{Isolated scattering number for interval graphs}

Interval graphs are a large class of graphs and important in modelling useful networks [10]. This section tries to compute the isolated scattering number for interval graphs, and proves that isolated scattering number can be computed in polynomial time for interval graphs. First, we give the definition of an interval graph.

Definition 6 ([10]). An undirected graph $\mathrm{G}$ is called an interval graph if its vertices can be put into one to one correspondence with a set of intervals $\ell$ of a linearly ordered set (like the real line) such that two vertices are connected by an edge if and only if their corresponding intervals have nonempty intersection. We call $\ell$ an interval representation for $\mathrm{G}$.

Example 7. Figure 2 shows the interval representation of an interval graph G. 
Figure 2: An interval graph $G$ and an interval representation for it
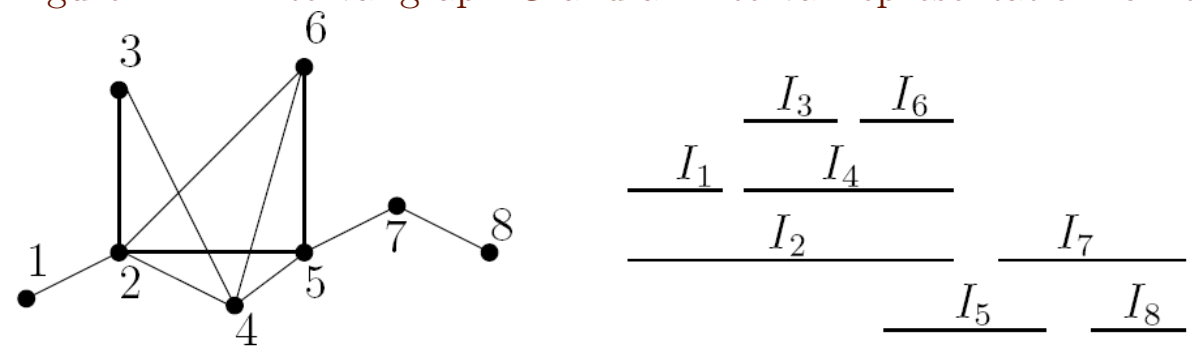

Interval graphs are a well-known family of perfect graphs with plenty of nice structural properties [10].

Interval graphs arise in the process of modelling many real life situations, especially those involving time dependencies or other restrictions that are linear in nature. This graph and various subclasses thereof arise in diverse areas such as archeology, molecular biology, sociology, genetics, traffic planning, very large-scale integration design, circuit routing, psychology, scheduling, transportation. Recently, interval graphs have found applications in protein sequencing [12], macro substitution [8], circuit routine [20], file organization [5], job scheduling [5], routing of two points nets [12], and so on. In addition to these, interval graphs have been studied intensely from both the theoretical and algorithmic point of view. Kratsch et al. [15] computed the toughness and the scattering number for interval and other graphs. Li and Li [18] proved the problem of computing the neighbour scattering number of an interval graph can be solved in polynomial time. Broersma et al. [4] gave linear-time algorithms for computing the scattering number and Hamilton-connectivity of interval graphs. In this section, we prove that there exists polynomial time algorithm for computing isolated scattering number of an interval graph.

The following characterisation was given by Gilmore and Hoffman [9].

Lemma 8 ([10]). Any induced subgraph of an interval graph is an interval graph. 
Lemma $9([3])$. An interval graph with order $\mathfrak{n}$ and size $\mathfrak{m}$ can be recognised in $\mathrm{O}(\mathrm{m}+\mathrm{n})$ time.

Lemma 10 ([10]). A triangulated graph on $\mathrm{n}$ vertices has at most $\mathrm{n}$ maximal cliques, with equality if and only if the graph has no edges.

Lemma 11 ([9]). A graph $\mathrm{G}$ is an interval graph if and only if the maximal cliques of $\mathrm{G}$ can be linearly ordered, such that, for every vertex $v$ of $\mathrm{G}$, the maximal cliques containing $v$ occur consecutively.

Such a linear ordering of the maximal cliques of an interval graph is said to be a consecutive clique arrangement. Notice that interval graphs are triangulated graphs, and by Lemma 10 we know that an interval graph with $n$ vertices has at most $n$ maximal cliques. Booth and Lueker [3] give a linear time recognition algorithm for interval graphs and the algorithm also computes a consecutive clique arrangement of the input graph if it is an interval graph.

Using Lemma 4, we identify the minimal separators of an interval graph G.

Lemma $12([15])$. Let $\mathrm{G}$ be an interval graph and let $\mathrm{A}_{1}, \mathrm{~A}_{2}, \ldots, \mathrm{A}_{\mathrm{t}}, \mathrm{t} \leqslant \mathrm{n}$, be a consecutive clique arrangement of $\mathrm{G}$. Then the set of all minimal separators of $G$ consists of vertex set $S_{p}=A_{p} \cap A_{p+1}, p \in\{1,2, \ldots, t-1\}$.

Hence an interval graph $G=(V, E)$ on $n$ vertices has at most $n$ minimal separators.

Definition $13([15])$. Let $\mathrm{G}$ be an interval graph with consecutive clique arrangement $A_{1}, A_{2}, \ldots, A_{t}$. We define $A_{0}=A_{t+1}=\emptyset$. For all $l, r$ with $1 \leqslant l \leqslant r \leqslant t$ we define $\mathcal{P}(l, r)=\left(\cup_{i=l}^{r} A_{i}\right)-\left(A_{l-1} \cup A_{r+1}\right)$. A set $\mathcal{P}(l, r), 1 \leqslant$ $\mathrm{l} \leqslant \mathrm{r} \leqslant \mathrm{t}$, is said to be a piece of $\mathrm{G}$ if $\mathcal{P}(\mathrm{l}, \mathrm{r}) \neq \emptyset$ and $\mathrm{G}[\mathcal{P}(\mathrm{l}, \mathrm{r})]$ is connected. Furthermore, $\mathrm{V}=\mathcal{P}(1, \mathrm{t})$ is a piece of $\mathrm{G}$ (even if $\mathrm{G}$ is disconnected).

Cliques in $\mathcal{P}(l, r)$ are listed in the same order as that they are listed in graph G. 
Lemma $14([15])$. Let $\mathrm{X}$ be a minimal separator of a connected subgraph $\mathrm{G}[\mathcal{P}(\mathrm{l}, \mathrm{r})], 1 \leqslant \mathrm{l} \leqslant \mathrm{r} \leqslant \mathrm{t}$. Then there exists a minimal separator $\mathrm{S}_{\mathrm{p}}$ of $\mathrm{G}$, $1 \leqslant p \leqslant r$, such that $X=S_{p} \cap \mathcal{P}(l, r)=S_{p}-\left(A_{l-1} \cup A_{r+1}\right)$. Moreover, every connected component of $\mathrm{G}[\mathcal{P}(\mathrm{l}, \mathrm{r})-\mathrm{X}]$ is a piece of $\mathrm{G}$.

From the definition of piece of $\mathrm{G}$, there are essentially two different types of pieces in an interval graph. A piece is called complete if it induces a complete graph and it is called incomplete otherwise. Pieces $\mathcal{P}(l, l)$ are complete. Furthermore, a piece $\mathcal{P}(l, r), l<r$, may also be complete. And for every complete piece $\mathrm{G}[\mathcal{P}(\mathrm{l}, \mathrm{r})], \mathrm{l}<\mathrm{r}$,

$$
\operatorname{isc}(\mathrm{G}[\mathcal{P}(l, r)])=2-|\mathrm{V}(\mathrm{G}[\mathcal{P}(l, r)])| .
$$

The incomplete piece $G[\mathcal{P}(l, r)], 1 \leqslant l \leqslant r \leqslant t$, has minimal separators and so

$$
\operatorname{isc}(G[\mathcal{P}(l, r)])=\max \left\{\sum_{i=k+1}^{t} \max \left[\operatorname{isc}\left(G\left[P_{i}\right]\right), 0\right]-\left|S_{\mathfrak{p}} \cap \mathcal{P}(l, r)\right|+k\right\},
$$

where the maximum is taken over all $S_{p} \cap \mathcal{P}(l, r), p \in\{1,2, \ldots, r-1\}$, that are minimal separators of $G[\mathcal{P}(l, r)], P_{1}, P_{2}, \ldots, P_{k}$ are the components of $G\left[\mathcal{P}(l, r)-S_{p}\right]$ which are isolated vertices, and $P_{k+1}, P_{k+2}, \ldots, P_{t}$ are the connected components of $G\left[\mathcal{P}(l, r)-S_{p}\right]$ which are not isolated vertices

Let $G$ be an interval graph. If $G$ is complete, then $\operatorname{isc}(G)=2-|V(G)|$. Otherwise the 'dynamic programming on pieces' works with the following steps.

1. Compute a consecutive clique arrangement $A_{1}, A_{2}, \ldots, A_{t}$ of $G$, then compute $l(v)=\min \left\{k: v \in A_{k}\right\}$ and $r(v)=\max \left\{k: v \in A_{k}\right\}$ for every $v \in V$, and then compute all minimal separators $S_{p}=A_{p} \cap A_{p-1}$, $p \in\{1,2, \ldots, t-1\}$.

2. For all $l, r$ with $1 \leqslant l \leqslant r \leqslant t$ compute the vertex set $\mathcal{P}(l, r)$, mark $(l, r)$ 'empty' if $\mathcal{P}(l, r)=\emptyset$ and mark $(l, r)$ 'complete' if $\mathcal{P}(l, r) \neq \emptyset$ and $\mathrm{G}[\mathcal{P}(l, r)]$ is a complete induced graph. 
3. For all non-marked tuples $(l, r)$ check whether $G[\mathcal{P}(l, r)]$ is connected. If so, mark $(l, r)$ 'incomplete'. Else, mark $(l, r)$ 'disconnected', and then compute the components $P_{j}=\mathcal{P}\left(l_{j}, r_{j}\right), 1 \leqslant j \leqslant k$, of $G[\mathcal{P}(l, r)]$ and store $\left(l_{1}, r_{1}\right),\left(l_{2}, r_{2}\right), \ldots,\left(l_{k}, r_{k}\right)$ in a linked list with a pointer from $(l, r)$ to the head of this list.

4. For all marked 'incomplete' tuples $(l, r), 1 \leqslant l \leqslant r \leqslant t$, compute the components $P_{j}=\mathcal{P}\left(l_{j}, r_{j}\right), 1 \leqslant j \leqslant k$, of $G\left[\mathcal{P}(l, r)-S_{p}\right]$, and then check whether $S_{p} \cap \mathcal{P}(l, r)$, is a minimal separator of $G[\mathcal{P}(l, r)]$, and if so, mark $(p, l, r)$ 'minimal', store $\left(l_{1}, r_{1}\right),\left(l_{2}, r_{2}\right), \ldots,\left(l_{k}, r_{k}\right)$ in a linked list with a pointer from $(p, l, r)$ to the head of this list and compute $\left|S_{p} \cap \mathcal{P}(l, r)\right|$.

5. For every pair $(l, r)$ marked 'complete' compute isc $(G[\mathcal{P}(l, r)])$ according to equation (1).

6. For $\mathrm{d}:=1$ to $t$, and for $l:=1$ to $t-d$, if $(l, l+d)$ is marked 'incomplete', compute isc $(\mathrm{G}[\mathcal{P}(l, l+d)])$ according to equation (2).

7. Output isc(G) $=\operatorname{isc}(G[\mathcal{P}(1, t)])$.

Theorem 15. The above algorithm outputs the isolated scattering number for an interval graph of order $\mathrm{n}$ with time complexity $\mathrm{O}\left(\mathrm{n}^{4}\right)$.

Proof: The correctness of this algorithm follows from Theorem 5 and Lemma 14. Steps 1, 2, 5 and 7 can be done in time $\mathrm{O}\left(\mathrm{n}^{4}\right)$ in a straightforward manner. In Step 3, testing connectedness and computing the components can be done by an $\mathrm{O}(n+m)$ algorithm for at most $n^{2}$ graphs $G[\mathcal{P}(l, r)]$. If $G[\mathcal{P}(l, r)]$ is disconnected and $P_{j}$ is a component, then $P_{j}=\mathcal{P}\left(l_{j}, r_{j}\right)$, $1 \leqslant j \leqslant k$, with $l_{j}=\min \left\{l(v): v \in P_{j}\right\}$ and $r_{j}=\max \left\{r(v): v \in P_{j}\right\}$ which can be computed in time $\mathrm{O}(\mathrm{n})$. Hence, Step 3 can be done in time $\mathrm{O}\left(\mathrm{n}^{4}\right)$.

Step 4 has to be executed for at most $n^{3}$ triples $(p, l, r)$ with $l \leqslant p<r$. If $\mathcal{P}(l, r)-S_{p} \neq \emptyset$, then the set of components of $G\left[\mathcal{P}(l, r)-S_{p}\right]$ is equal to the union of the set of components of $G[\mathcal{P}(l, p)]$ and of the set of components 
of $\mathrm{G}[\mathcal{P}(p+1, r)]$ or it is equal to one of these sets. Hence, the components of are $\mathrm{G}\left[\mathcal{P}(l, r)-S_{p}\right]$ are computed by using the marks of $(l, p)$ and $(p+1, r)$, namely, if the mark is 'complete' or 'incomplete', then $(l, p)$ and $(p+1, r)$, respectively, are stored and if the mark is 'disconnected', then the corresponding linked list is added. Thus the linked list of $(p, l, r)$ can be computed in time $\mathrm{O}(\mathrm{n})$. Since $S_{p} \cap \mathcal{P}(l, r)$ is a minimal separator of $G[\mathcal{P}(l, r)]$ if and only if there are at least two different connected components in the list of $(p, l, r)$ such that every vertex of $S_{p} \cap \mathcal{P}(l, r)$ has a neighbour in both of these components (Lemma 4). Because of the properties of a consecutive clique arrangement it suffices to check that two components $P_{j}$ of $G[\mathcal{P}(l, p)]$ with the two largest values of $r_{j}$ and the two components of $P_{j}$ of $G[\mathcal{P}(p+1, r)]$ with the two smaller values of $l_{j}$ (if they exist). This can be done in time $O(n)$. Hence Step 4 needs time $\mathrm{O}\left(\mathrm{n}^{4}\right)$.

Step 6 requires the evaluation of the right-hand side of equation (2) for at most $n^{2}$ pairs $(l, l+d)$. For every $p$ with $l \leqslant p<l+d$ and $(p, l, l+d)$ marked 'minimal' the components $P_{j}$ of $G\left[\mathcal{P}(l, l+d)-S_{p}\right]$ can be obtained in time $\mathrm{O}(n)$ from the linked list of $(p, l, l+d)$. Each of the at most $n$ values isc $\left(G\left[P_{i}\right]\right)$ can be determined in constant time by table look-up since the isolated scattering numbers of smaller pieces are already known. Thus $\sum_{i=1}^{k} \max \left\{\operatorname{isc}\left(G\left[G\left[P_{i}\right]\right), 1\right\}-\left|S_{p} \cap \mathcal{P}(l, l+d)\right|\right.$ can be evaluated in time $O(n)$. Consequently, Step 6 of the algorithm can be done in time $\mathrm{O}\left(\mathrm{n}^{4}\right)$.

\section{Conclusion}

Network vulnerability is an important issue in the area of distributed computing. Networks such as computer or communication networks are so designed that they do not easily get disrupted under external attack and, moreover, these are easily reconstructed when they do get disrupted. Most of the early work in this area takes a probabilistic approach to the problem. However, sometimes it is important to incorporate subjective vulnerability estimates 
into the measure. In this paper, we address a comparatively better parameter, the isolated scattering number, which measures the vulnerability of networks, and we give a polynomial time algorithm to compute the isolated scattering number of interval graphs, a subclass of co-comparability graphs. As a useful parameter, there are many problems remaining unsolved. One interesting problem is to find isolated scattering number conditions for the existence of certain factors in graphs.

Acknowledgements This work was supported by NSFC (No. 11471003 and 11401389), Zhejiang Provincial Natural Science Foundation of China(No. LY17A01 and by the China Scholarship Council (No. 201607910003). Especially, the authors are very thankful to anonymous referees for their constructive suggestions and critical comments, which led to this improved version.

\section{References}

[1] C. A. Barefoot, R. Entringer and H. Swart. Vulnerability in graphs-A comparative survey. J. Combin. Math. Combin. Comput. 1:12-22, 1987. https://www.researchgate.net/publication/266002676 E83

[2] J. A. Bondy and U. S. R. Murty. Graph Theory with Applications. Macmillan, London and Elsevier, New york, 1976. http:

//101.96.10.59/www.iro.umontreal.ca/ ^hahn/IFT3545/GTWA.pdf $\mathrm{E} 83$

[3] K. S. Booth and G. S. Lueker. Testing for the consecutive ones property, interval graphs, and graph planarity using PQ-tree algorithms. $J$. Comput. System Sci. 13(3):335-379, 1976. doi:10.1016/S0022-0000(76)80045-1 E90

[4] H. Broersma, J. Fiala, P. Golovach, T. Kaiser, D. Paulusma, A. Proskurowski. Linear-time algorithms for scattering number and 
hamilton-connectivity of interval graphs. J Graph Theory 79(4): 282-299, 2015. doi:10.1002/jgt.21832 E89

[5] M. C. Carlisle, E. L. Loyd. On the k-coloring of intervals. LNCS 497: 90-101, 1991. doi:10.1016/0166-218X(95)80003-M E89

[6] V. Chvatal. Tough graphs and Hamiltonian circuits. Discrete Mathematics 5:215-228, 1973. doi:10.1016/j.disc.2006.03.011 E83

[7] M. Cozzens, D. Moazzami and S. Stueckle. The tenacity of a graph. Proc. 7th International Conference on the Theory and Applications of Graphs, Wiley, New York, 1111-1122, 1995. http: //101.96.10.59/www.iro.umontreal.ca/ 〜hahn/IFT3545/GTWA.pdf $\mathrm{E} 83$

[8] J. Fabri. Automatic Storage Optimization. UMI Press Ann Arbor, MI, 1982. doi:10.1145/989393.989398 E89

[9] P. C. Gilmore and A. J. Hoffman. A characterization of comparability graphs and of interval graphs. Canadian J. Math. 16(99):539-548, 1964. doi:10.1142/97898127969360006 E89, E90

[10] M. C. Golumbic. Algorithmic Graph Theory and Perfect Graphs. Academic Press, 1980. doi:10.1007/BF00390110 E88, E89, E90

[11] H. A. Jung. On maximal circuits in finite graphs. Ann Discrete Math. 3:129-144, 1978. doi:10.1016/S0167-5060(08)70503-X E83

[12] J. R. Jungck, O. Dick, and A. G. Dick. Computer assisted sequencing, interval graphs and molecular evolution. Biosystem 15:259-273, 1982. doi:10.1016/0303-2647(82)90010-7 E89

[13] T. Kloks and D. Kratschz. Listing all minimal separators of a graph. SIAM J. Comput. 27(3):605-613, 1998. doi:10.1137/S009753979427087X $\mathrm{E} 85$

[14] T. Kloks, D. Kratsch and J. Spinrad. Tree-width and path-width of co-comparability graphs of bounded dimension. Computing Science 
Note. Eindhoven University of Technology, Eindhoven, The Netherlands. 93-46. https:

alexandria.tue.nl/extra1/wskrap/publichtml/9313455.pdf E85

[15] D. Kratsch, T. Klocks and H. Müller. Computing the toughness and the scattering number for interval and other graphs. IRISA resarch report. France, 1994.

https://www.researchgate.net/publication/2646060 E89, E90, E91

[16] F. W. Li. On isolated rupture degree of graphs. Utilitas Mathematica 96: 33-47, 2015.

https://www.researchgate.net/publication/292526797 E83, E84

[17] F. W. Li. Isolated rupture degree of trees and gear graphs. Neural Network World 25(3): 287-300, 2015. doi:10.14311/NNW.2015.25.015 $\mathrm{E} 83$

[18] F. W. Li and X. L. Li. Neighbor-scattering number can be computed in polynomial time for interval graphs. Computers and Mathematics with Applications 54(5):679-686, 2007. doi:10.1016/j.camwa.2007.02.006 E89

[19] Y. K. Li, S. G. Zhang and X. L. Li. Rupture degree of graphs. Int. J. Comput. Math. 82(7):793-803, 2005. doi:10.1080/00207160412331336062 E83

[20] T. Ohtsuki, H. Mori, Khu. E. S., T. Kashiwabara, T. Fujisawa. One dimensional logic gate assignment and interval graph. IEEE Trans. Circuits and Systems 26:675-684, 1979. doi:10.1109/TCS.1979.1084695 E89

[21] S. Y. Wang, Y. X. Yang, S. W. Lin, J. Li and Z. M. Hu. The isolated scattering number of graphs. Acta Math. Sinica (in Chinese) 54(5):861-874, 2011. http:

//en.cnki.com.cn/Article_en/CJFDTotal-SXXB201105015.htm E84 


\section{Author addresses}

1. Fengwei Li, Department of Mathematics, Shaoxing University, Shaoxing, Zhejiang 312000, P.R. China.

mailto:fengwei.li@hotmail.com orcid:0000-0003-2791-1683

2. Qingfang Ye, Department of Mathematics, Shaoxing University, Shaoxing, Zhejiang 312000, P.R. China.

mailto: fqy-y@163. com

orcid:0000-0001-9000-2330

3. Yuefang Sun, Department of Mathematics, Shaoxing University, Shaoxing, Zhejiang 312000, P.R. China.

mailto:yfsun2013@gmail.com orcid:0000-0002-7515-8481 